\title{
LE TUBAGE GASTRIQUE CHEZ LES POISSONS : MISE AU POINT D'UN EQUIPEMENT ET TEST CHEZ LE CHABOT Cottus gobio L.
}

\author{
P. GaUdin, E. MARTIN, L. CaILlere \\ Laboratoire d'Etude des Rythmes et Comportement \\ Structures et Mécanismes \\ E.R.A. - C.N.R.S. $n^{\circ} 849$ - Université LYON | - 69622 VILLEURBANNE CEDEX
}

\section{RESUME}

Un système de vidange stomacale a été mis au point dans le but de préciser le régime alimentaire d'une petite population de chabots (Cottus gobio L.). Le matériel et les prıncipes d'utilisation sont décrits. La méthode a été testée au laboratoire et sur le terrain. L'expérimentation réalisée au laboratoire montre que :

1 - l'efficacité de la vidange stomacale est très satisfaisante $(98,2 \%)$,

2 - le taux de réalimentation est très élevè 24 heures après la vidange,

3 - la survie, un mois après la vidange, est de $100 \%$

Dans les conditions de pêche électrique, la méthode s'est avérée efficace, la survie au bout de 24 heures étant voisine de celle constatée après une pêche électrique classique.

Cette méthode est pratique (le temps de manipulation, par poisson, n'excède pas 1,5 minute), et permet une bonne approche du régime alimentaire du chabot dans les conditions naturelles, sans que le poisson soit sacririé. Son application entraine également un gain de temps appréciable par rapport aux méthodes traditionnelles d'étude du régime alimentaire des Poissons.

\section{SUMMARY}

A technique for stomach sampling was developed for studying the feeding habits of the Sculpin Cottus gobio L. The equipment and the steps of the method were described, both in laboratory and field conditions.

The efficiency of gastric tubing (number of preys collected with respect to that of ingested preys) is very good $(98,2 \%)$, and the survival of fishes too (100\%) within the month following the experimentation in the laboratory. This method seems to be handy (the manipulation of a fish did not exceed 1,5 minute) and enables a good approach, in natural conditions, of the feeding habits of the Sculpin.

\section{INTRODUCTION}

L'étude classique du régime alimentaire des poissons présente l'inconvénient majeur d'entrainer la mort d'un grand nombre d'individus. En effet, de nombreux poissons doivent être prélevés puis tués afin d'extraire le contenu du tube digestif qui sera alors analysé.

Si ce prélèvement peut parfois être bien toléré par le milieu, il provoque généralement des déséquilibres dont l'importance dépend de la' densité de l'espèce étudiée et du degré d'isolement du milieu où le prélèvement est effectué.

L'ètude que nous menons sur la prédation du Chabot (Cottus gobio L.) en particulier sur le frai de truite (Salmo trutta L.), se faisant, d'une part dans un petit ruisseau pépinière et d'autre part sur une faible population de poissons (une centaine d'individus), il nous a paru indispensable de prélever les contenus digestifs des poissons sans les sacrifier. C'est pourquoi nous avons mis au point un système de vidange gastrique proche, dans ses principes, de celui proposé par SEABURG en 1957.

Cependant, avant d'utiliser ce matériel sur le terrain il était nécessaire de tester son efficacité et son influence sur la survie des poissons, au laboratoire.

\section{MATERIEL ET METHODE}

\section{A - Matériel}

L'équipement mis au point afin de prélever le contenu stomacal des poissons comprend, d'une part une série de quatre embouts adaptés à la morphologie des poissons 
étudiés, d'autre part une tablette rassemblant les divers éléments nécessaires au déroulement de l'opération.

\section{LES EMBOUTTS}

Quatre embouts de tailles différentes ont été réalisés. Chacun est constitué de deux tubes de diamètre différent soudés l'un à l'autre. L'eau est injectée dans l'estomac par le petit tube. Le mélange eau + proies ressort par le gros tube (fig. 1):

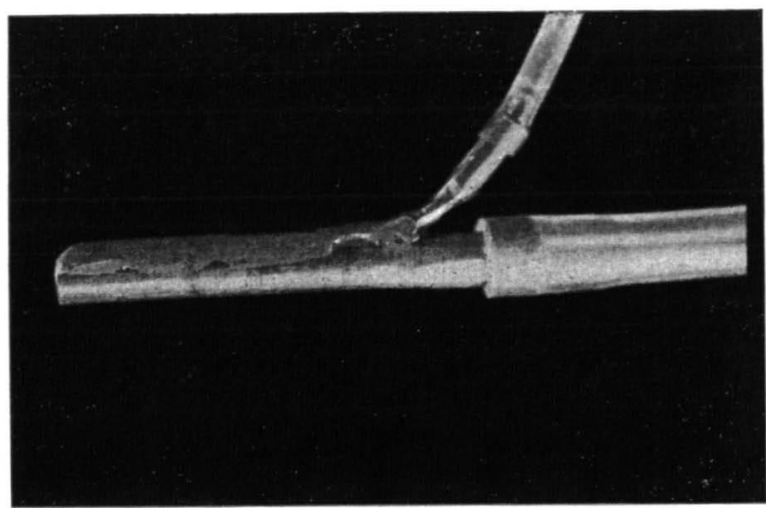

Figure 1 : Embout vu de profil

Pour la fabíication des quatre embouts nous disposions de 6 diamètres de tubes dont les caractèristiques sont données dans le tableau 1

Tableau 1 : Caractéristiques des tubes utilisés pour la fabrication des différents embouts.

\begin{tabular}{|c|c|c|}
\hline$n^{\circ}$ du tube & diamètre, intérieur $(\mathrm{mm})$ & diamètre: extérieur $(\mathrm{mm})$ \\
\hline I & 1 & 2 \\
II & 2 & 3 \\
III & 3 & 4 \\
IV & 4 & 5 \\
V & 5 & 6 \\
VI & 6 & 7 \\
\hline
\end{tabular}

L'ensemble est alors poli au pápiér de verre très fin, en particulier au niveau de l'extrémité qui ne doit pas provoquer de lésion au moment de l'introduction dans l'œsophage du poisson.

Les caractéristiques des quatre embouts obtenuś sont donnèes dans le tableau 2.

Tableau 2 : Caractéristiques des différents embouts.

\begin{tabular}{|c|c|c|c|c|c|c|}
\hline \multirow{2}{*}{$\begin{array}{l}N^{\circ} \text { de } \\
\text { l'embout }\end{array}$} & \multicolumn{2}{|c|}{ tubes utilisés } & \multicolumn{2}{|c|}{$\begin{array}{c}\text { diamètre } \\
\text { extérieur ( } \mathrm{mm})\end{array}$} & \multirow{2}{*}{$\begin{array}{r}\text { diamètre } \\
\text { - intérieur } \\
(\mathrm{mm})\end{array}$} & \multirow{2}{*}{$\begin{array}{l}\text { longueur } \\
(\mathrm{mm})\end{array}$} \\
\hline & arrivée d'eau & $\begin{array}{l}\text { sortie eau } \\
+ \text { proies }\end{array}$ & $\min i$ & maxi. & & \\
\hline 1 & 1 & III & 4 & 4,5 & 3 & 52 \\
\hline 2 & 1 & IV & 5,2 & 6.1 & 4 & 49 \\
\hline 3 & II & $v$ & 6.2 & 7.6 & 5 & 58 \\
\hline 4 & II & VI & 7.3 & 8,5 & 6 & 58 \\
\hline
\end{tabular}

Précisons que l'embout $n^{\circ} 4$, d'un diamètre extérieur élevé, n'a jusqu'à présent pu être utilisé sur les chabots, aucun spécimen de taille suffisamment importante n'ayant étè capturé dans le ruisseau étudié. 


\section{LA TABLETTE}

\section{Description (fig. 2)}

Là table de contention (1) sert au maintien du poisson pendant l'opération

L'embout (2) est relié d'une part à un système d'injection de l'eau, d'autre part à un pilulier permettant de recueillir le mélange eau + proies.

L'eau servant à nettoyer l'estomac est stockée dans un réservoir (3). Elle est injectée par une poire (4) munie d'une valve à chaque extrémité, ce qui permet de produire le courant d'eau dans une seule direction.

Le pilulier dans lequel est recueilli le mélange eau + proies (5) est muni d'un bouchon (6) traversé par deux tuyaux : I'un (7) canalise les proies extraites de l'estomac, l'autre (8) assure, par siphonage, l'évacuation de l'eau à l'extérieur: les particules alimentaires étant retenues dans le pilulier par un grillage très fin placé au dèpari du tube (fig. 3).

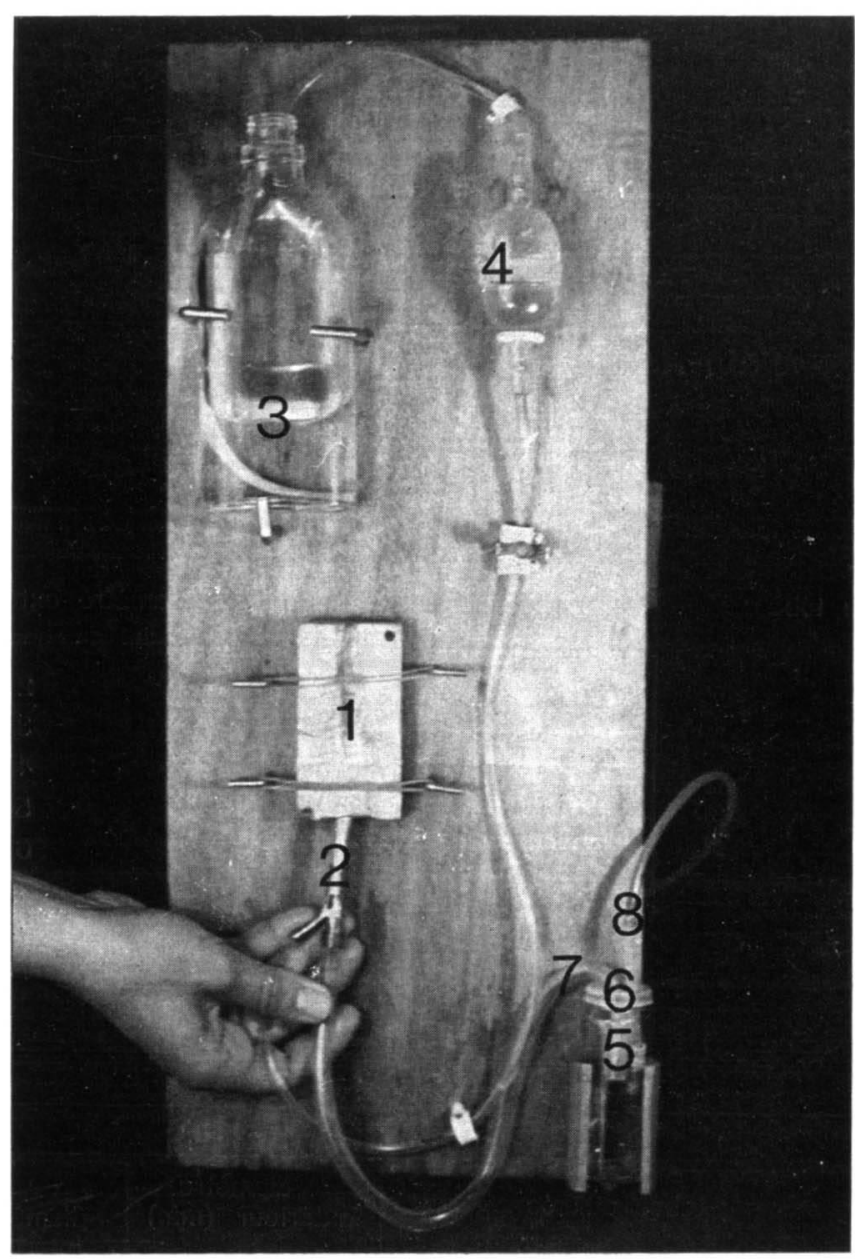

Figure 2 : Vue d'ensemble de la tablette utilisée pour les vidanges stomacales.

1 - table de contention

2 - embout

3 - réservoir

4 - poire

5 - pilulier

6 - bouchon

7 - tuyau par lequel arrive le mélange eau + proies

8 - tuyau d'évacuation de l'excès d'eau 


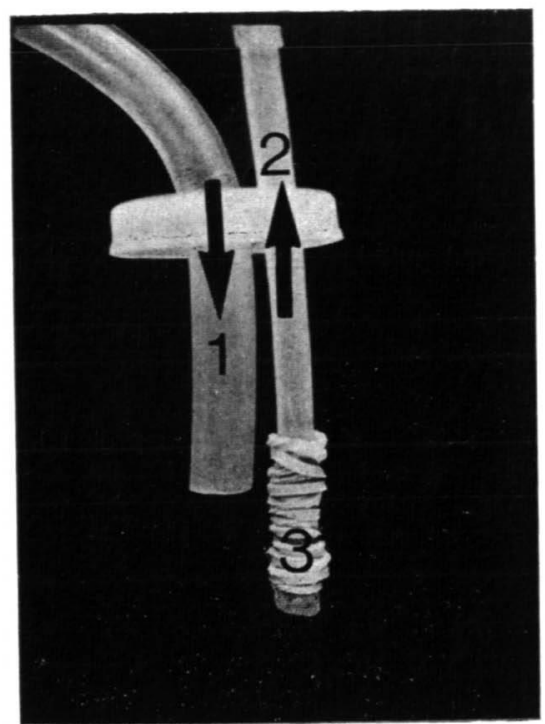

Figure 3 : Détail $n^{\circ} 6$ de la figure 2 : bouchon du pilulier

1 - Arrivée du mélange eau + proies

2 - Evacuation de l'eau

3 - Tamis (vide de maille $=0,2 \mathrm{~mm}$ )

\section{B - Méthode}

\section{OPERATION}

La planche est inclinée à $45^{\circ}$.

Le poisson, anesthésié au phénoxyéthanol $(0,5 \mathrm{ml} / \mathrm{l})$, est placé sur la table de contention, la tête en bas.

L'embout est alors introduit dans la bouche du poisson. Au passage de la ceinture scapulaire une pression légère mais soutenue permet l'introduction de celui-ci dans l'œsophage.

Sachant que la partie antérieure de l'estomac du chabot est située à l'aplomp de l'insertion des nageoires pectorales, le guidage de l'embout jusqu'à l'estomac peut être effectué avec précision.

L'eau peut alors être injectée, de préférence par petits jets rapides. Si l'embout est cor'éctement placé, l'abdomen se dilate au moment de l'injecțon.

L'opération terminée, le roisson est immédiatement remis à l'eau. Le contenu digestif est alors fixé par de l'alcool ou du formol dans le pilulier où il a été prélevé. Un pilulier identique est ensuite installé à la place du précédent': la vidange stomacale d'un autre poisson peut alors être réalisée. Si l'embout est insuffisamment enfoncé ou obturé par une proie volumineuse, de l'eau peut s'écouler par la bouche le long te l'embout. II sulfit alors de retirer celui-ci, de le déboucher, et de répéter l'opération.

\section{TEST DE LA METHODE AU LABORATOIRE}

Afin d'évaluer l'efficacité de la vidange gastrique ainsi que le stress qu'elle provoque chez le poisson testé, nous avons procédé comme suit :

1. Le poissọn est soumis à un jeûne de $72 \mathrm{H}\left(\right.$ à $\left.15^{\circ} \mathrm{C}\right)$, ce laps de temps correspondant à la déplétion de l'estomac chez le chabot (WESTERN, 1971).

2. Après le jeûne, 15 gammares sont proposès au poisson pendant une demi-heure environ. En effet, quand un chabot a été soumis au jeûne, il capture généralement les proies dans la demi-heure qui suit leur introduction (GAUDIN, 1979).

3. Après une demi-heure, le poisson est prélevé, anesthésié, mesuré et pesé; la vidange est effectuée et la taille de l'embout utilisé est notée. 
4. Après cette opération, on compte le nombre de proies contenues dans le pilulier et le nombre de proies restant dans le bac où était le chabot. On peut ainsi savoir si la vidange a été totale.

5. Le chabot est ensuite placé dans un bac contenant des proies (gammares et/ou larves de chironomes).

6. 24 heures après la première vidange stomacale, 'opération est répétée afin de savoir si le chabot s'est réalimenté.

7. Pendant la semaine qui suit le test, la survie de chaque chabot est quotidiennement contrôlée.

8. Les poissons sont ensuite rassemblés dans un bac afin de contrôler globalement leur survie pendant au moins un mois après l'opération.

Nous pouvons donc définir:

- L'efficacité : la vidange est efficace si toutes les proies ingérées par le chabot avant la première vidange stomacale sont prélevées au cours de l'opération.

- La réalimentation : on considère qu'il $y$ a réalimentation si des proies sont extraites de l'estomac au cours de la deuxième vidange $(24 \mathrm{H}$ après la premiere).

- La survie.

\section{TEST DE LA METHODE SUR LE TERRAIN}

Cette méthode a été utilisée deux fois au cours de pêches effectuées sur un petit affluent du Tillery qui se jette lui-même dans le Suran au niveau de la commune de Germagnat (Ain).

Si le but premier de ces pêches n'était pas le test de la méthode, nous en avons cependant profité pour noter les limites de taille et de poids dans lesquelles chaque embout était utilisé.

Par ailleurs, nous avons également relevé la mortalité : une heure après l'opération pour la première pêche et 24 heures après l'opération pour la deuxième pêche.

\section{RESULTATS}

\section{A - Au laboratoire}

Un premier test a été effectué sur 44 poissons. Quatre d'entre eux; ne s'étant jamais alimentés, n'ont pas été pris en compte dans le traitement des résultats qui porteront donc sur 40 individus.

Un deuxième test a èté effectué sur 16 des 44 poissons déjà testés. Tous se sont alimentés au moins une fois.

\section{EFFICACITE}

Une seule vidange ayant été incorrecte sur l'ensemble des deux tests, le pourcentage d'efficacité est donc de $98,2 \%$ (Tableau 3).

\section{REALIMENTATION}

Pour le premier test (Tableau 3), quatre poissons ne se sont pas réalimentés 24 heures après la vidange. Pour le deuxième test tous se sont réalimentés. Donc sur 56 poissons $92,9 \%$ se sont réalimentés 24 heures après la vidange.

3. SURVIE

Dans les deux tests les taux de survie contrôlés sont de $100 \%$ (Tableau 3)

Tableau 3 : Résultats des deux séries d'expérimentations au laboratoire.

\begin{tabular}{|l|c|c|}
\hline Nombre de poissons testés & $1^{\text {er } \text { test }}$ & $2^{\text {o test }}$ \\
\hline$\%$ poissons alimentés & 40 & 16 \\
\hline$\%$ poissons réalimentés après $24 \mathrm{~h}$. & $80 \%$ & $69 \%$ \\
\hline$\%$ vidange incorrecte & $90 \%$ & $100 \%$ \\
\hline Survie après une semaine & $2,5 \%$ & $0 \%$ \\
\hline Survie après au moins un mois & $100 \%$ & $100 \%$ \\
\hline
\end{tabular}




\section{TAILLE DES EMBOUTS} bleau 4).

Chaque emboui peut être utilisé dans certaines limites de taille et de poids (Ta-

Tableau 4 : Limites d'utilisation des différen:s embouts dans les conditions de laboratoire.

\begin{tabular}{|c|c|c|c|c|c|}
\hline \multirow[b]{2}{*}{ No de l'embout } & \multicolumn{2}{|c|}{ MINIMUM } & \multicolumn{2}{|c|}{ MAXIMUM } & \multirow{2}{*}{$\begin{array}{c}\text { Nbre poissons } \\
\text { testés }\end{array}$} \\
\hline & $\begin{array}{l}\text { Poids } \\
\text { (g) }\end{array}$ & $\begin{array}{l}\text { Taille } \\
(\mathrm{mm})\end{array}$ & $\begin{array}{l}\text { Poids } \\
\text { (g) }\end{array}$ & $\begin{array}{l}\text { Taille } \\
(\mathrm{mm})\end{array}$ & \\
\hline 1 & 4,2 & 73 & 6,7 & 82 & 6 \\
\hline 2 & 5 & 79 & 11,4 & 100 & 36 \\
\hline 3 & 10,6 & 94 & 12,5 & 99 & 2 \\
\hline
\end{tabular}

Tous les poissons que nous avions en élevage ayant pu être testés, la taille minimum donnée pour l'embout $n^{\circ} 1$ ne peut être considérés comme absolue; en effet, dans la mesure où nous disposerons de poissons de taille plus faiblé cette valeur pourra être précisée.

De même, deux poissons seulement ayant pu être vidangés avec l'embout $n^{\circ} 3$, les chiffres présentés n'ont qu'une valeur d'indication. Nous attendons également de disposer d'individus de plus grande taille pour préciser ces limites.

Nous pouvons également constater qu'il y a un recouvrement entre les données ayant trait au maximum pour un embout et au minimum pour l'embout de diamètre supérieur.

\section{DUREE DE LA MANIPULATION}

Durée de l'anesthésie

: $2 \mathrm{mn}$

Durée des mesures (poids, taille)

: $20 \mathrm{sec}$.

Durée de la vidange

: 45 sec. à $1 \mathrm{mn} .30$.

Le temps nécessaire pour effectuer une vidange est variable car la taille de l'embout choisi peut ne pas convenir à la morphologie du chabot testé, il est alors nécessaire de changer l'embout.

\section{B - Sur le terrain}

\section{SURVIE}

Lors de la première pêche, les 88 poissons testés ont été remis à l'eau après récupération de l'anesthésie. Un seul d'entre eux étant mort la survie est de $98,9 \%$

A la deuxième pêche, les 136 poissons testés ont été gardés en vivier durant les 24 heures suivant la. vidange. Cinq d'entre eux étant morts la survie est de $96,3 \%$.

\section{TAILLE DES EMBOUTS}

La limite inférieure de l'utilisation de l'embout $n^{\circ} 1$ a cette fois pu être déterminée (Tableaux 5 et 6). Cependant l'embout $n^{\circ} 3$ n'a pu être utilisé que sur un seul poisson; nous ne pouvons donc pas évaluer les limites d'utilisation de cet embout.

Nous constatons également un recouvrement entre les données ayant trait au maximum pour un embout et au r.inimum pour l'embout qui lui succède.

Tableau 5 : Limites d'utilisation des différents embouts sur le terrain (première pêche).

\begin{tabular}{|c|c|c|c|c|c|}
\hline \multirow{2}{*}{ No de l'embout } & \multicolumn{2}{|c|}{ MINIMUM } & \multicolumn{2}{|c|}{ MAXIMUM } & \multirow{2}{*}{$\begin{array}{c}\text { Nombre } \\
\text { poissons testés }\end{array}$} \\
\hline & Poids (g) & Taille $(\mathrm{mm})$ & Poids (g) & Taille (mm) & \\
\hline $\begin{array}{l}\text { Poissons non } \\
\text { vidangeables }\end{array}$ & - & - & 4. & 71 & 56 \\
\hline embout $n^{\circ} 1$ & 3,6 & 71 & 8,4 & 92 & 19 \\
\hline embout $n^{\circ} 2$ & 7,2 & 83 & 14,6 & 102 & 12 \\
\hline embout $n^{\circ} 3$ & 18,6 & 112 & - & - & 1 \\
\hline
\end{tabular}


Tableau 6 : Limites d'utilisation des différents embouts sur le terrain (deuxième pêche).

\begin{tabular}{|c|c|c|c|c|c|}
\hline \multirow{2}{*}{$N^{0}$ de l'embout } & \multicolumn{2}{|c|}{ MINIMUM } & \multicolumn{2}{|c|}{ MAXIMUM } & \multirow{2}{*}{$\begin{array}{c}\text { Nombre } \\
\text { poissons testès }\end{array}$} \\
\hline & Poids (g) & Taille (mm) & Poids (g) & Taille $(\mathrm{mm})$ & \\
\hline $\begin{array}{l}\text { Poissons non } \\
\text { vidangeables }\end{array}$ & - & - & 4,1 & 69 & 35 \\
\hline embout $n^{0} 1$ & 3,3 & 67 & 7,6 & 83 & 55 \\
\hline embout $n^{\circ} 2$ & 6,7 & 79 & 15 & 102 & 46 \\
\hline
\end{tabular}

\section{DISCUSSION}

Bien que l'efficacité n'ait été testée que dans les conditions de laboratoire, les résultats obtenus montrent que l'équipement mis au point et la méthode utilisée sont tout à fait adaptés. Nous pouvons donc considérer que chaque vidange nous permet d'extraire la totalité du contenu stomacal de chaque poisson.

Le test, au laboratoire, de la réalimentation après vidange montre que la plupart des poissons se sont à nouveau nourris. Les contraintes mécaniques subies par le poisson ne semblent donc pas perturber la prise alimentaire et l'ingestion des proies dans les heures suivant le test. Nous avons d'ailleurs pu occasionneliement constater que de nombreuses prises alimentaires s'effectuaient peu de temps après le réveil du poisson.

De plus, aucune mórtalité n'ayant été relevée pendant une longue période (supérieure à un mois), et les poissons ayant subi un deuxième test ayant tous présenté un comportement alimentaire normal, nous pouvons penser que le passage de l'embout dans l'csophage ne provoque aucune lésion importante. Par ailleurs, le pourcentage de mortalité sur le terrain est du même ordre de grandeur que celui obtenu généralement au cours d'une pêche électrique.

La figure 4 présente pour chaque embout les tailles de poissons pour lesquelles il peut être utilisé.

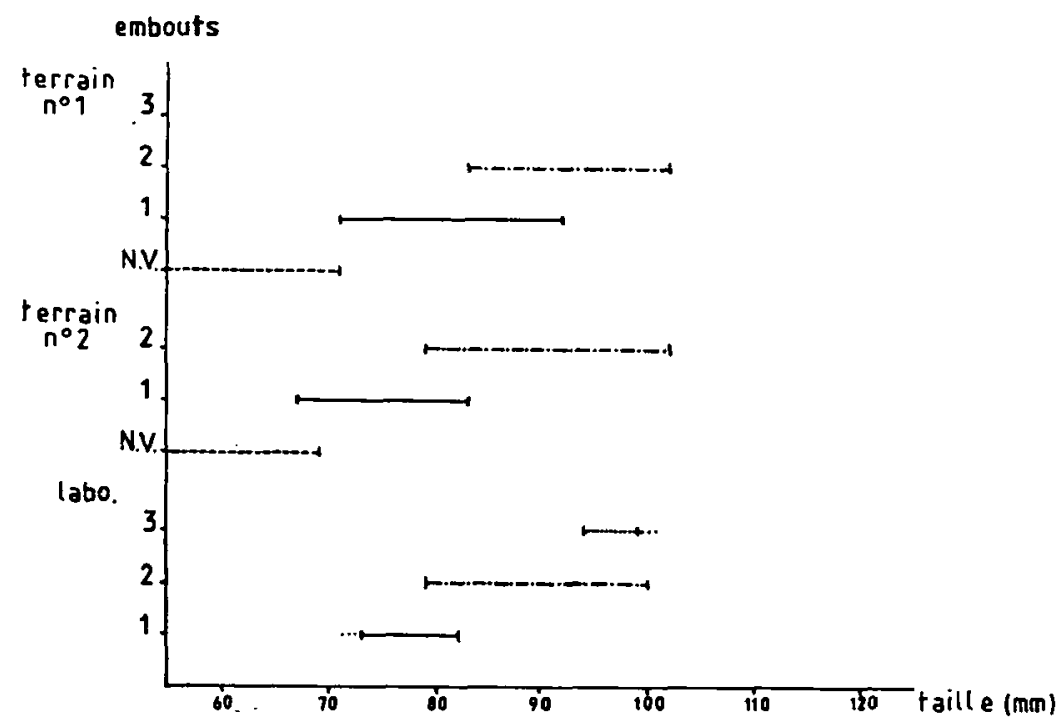

Figure 4 : Limites d'utilisation de chaque iype d'embout

- en abscisse : taille des poissons en $\mathrm{mm}$

- en ordonnée : 1.2.3. : types d'embout

N.V. : poissons non vidangeables 
Les recouvrements constatés sont dûs aux différences morphologiques et au sexe des individus : en effet, les mâles étant plus trapus que les femelles, ils présentent une ouverture buccale plus importante.

Nous pouvons également constater qu'au cours de la première pêche (terrain $n^{\circ} 1$ ), les limites d'utilisation des: embouts sont décalées vers des valeurs plus élevées. Comme cette pêche coincidait avec la première utilisation de l'équipement, nous craignions alors de provoquer des lésions de l'œsophage au niveau de la ceinture scapulaire: L'expérimentation qui a suivi, effectuée au laboratoire, nous a montré qu'une pression un peu plus importante ne provoquait aucune lésion. Nous avons donc pu, au cours de-la- deuxième pêche (terrain $n^{\circ} 2$ ) obtenir de meilleurs résultats tout à fait comparables à ceux obtenus dans les conditions de laboratoire.

Enfin, les chronométrages effectués au laboratoire et sur le terrain montrent que l'opération est suffisamment rapide pour permettre le prélèvement d'un nombre important de contenus digestifs au cours d'une seule pêche. De toute façon, le travail supplémentaire occasionné par la vidange stomacale est négligeable par rapport à celui demandé par la méthode généralement employée : 1) sur le terrain introduction des poissons dans un produit fiyateur, 2) au laboratoire dissection des poissoris, 3) nettoyage de l'estomac.

\section{$\checkmark$ CONCLUSION}

L'ensemble des résultats obtenus au cours de ce travail montre que le matériel mis au point est tout-à-fait adapté pour mener à bịen une étude du régime alimentaire du chabot.

Nous pensons qu'il serait intéressant de tester et de développer cette méthode en l'appliquant à d'autres espèces de Poissons. Un des intérêts de cette méthode est de pouvoir, à l'instar des études poursuivies actuellement sur le chabot, préciser le moment du nycthémère auquel a lieu la prise alimentaire, en se basant sur l'état de dégradation des proies ingérées. Cette méthode peut également permettre, a'une part des études longitüidinales de l'alimentation sur un même lot d'individus, d'autre part des études approfondies du régime alimentaire d'espèces à préserver à cause de leur importance économique (Truite) ou de leur rareté (Apron du Rhône). II faut cependant signaler que l'extraction des proies s'avère plus délicate chez les Poissons ichtyophages.

\section{Remerciements :}

Nous tenons à exprimer nos vifs remerciements, d'ine part au C.S.P. (Région Piscicole de Lyon) qui, en attribuant une bourse à E. MARTIN, a permis la réalisation d'une part importante de ces travaux, d'autre part à l'Association de Pêche et de Pisciculture - Les Pêcheurs Sportifs de l'Ain - qui nous a permis de mener à bien cette expérimentation dans le ruisseau de Tillery.

\section{BIBLIOGRAPHIE}

GAUDIN (P.), 1979. Données préliminaires à l'étude du comportement prédateur de Cottus gobio L. (Poisson). D.E.A. Univ. Lyon I.

SEABURG (K.G.), 1957. A stomach sampler for live fish. The progressive fish culturist, 19 (3), 137-139.

'NESTERN (I.R.H.), 1971. Feeding and digestion in two cottid fishes, the freshwater Cottus gobio and marine Enophrys bubalis. J. fish. biol. 3, 225-246. 